\title{
Shared Identity, Says Who? How Diverse Organizations Interact in Entrepreneurial Ecosystems
}

\begin{abstract}
Entrepreneurial ecosystems are comprised of a diverse and interacting set of organizations which aim to support and develop new ventures and their surrounding regions. Although prior research has celebrated the diversity of these support organizations as a necessary enabler of entrepreneurial capacity in a region, such diversity can at times introduce liabilities given different motives and interests. In this study, we explore how different organizations orient toward such diversity, thereby enabling or constraining the capacity for collaboration across ostensible divisions. Through an inductive case analysis of 15 organizations, we surface an inductive model of how leaders' perceptions of a regional ecosystem identity combine with their organizations' identity orientation to influence their interactions across the ecosystem. Based on this model, we propose a typology of organizational sponsorship, which characterizes organizations as either builders, partners, participants, or bridgers. This model and typology extends our understanding of the differences between organizational sponsors within entrepreneurial ecosystems, while bridging the study of organizational identity, categories and ecosystems.
\end{abstract}

Keywords: Entrepreneurial ecosystem, collective identity, organizational identity orientation, category buffering, regions 


\section{Shared Identity, Says Who? How Diverse Organizations Interact in Entrepreneurial Ecosystems}

\section{INTRODUCTION}

Entrepreneurial ecosystems are regions where actors, institutions, and support organizations collectively provide entrepreneurs with resources to facilitate the growth of new ventures (Autio, Nambisan, Thomas, \& Wright, 2018; Thompson, Purdy, \& Ventresca, 2018). These regions and the organizations established within them are important and celebrated in the emergence and success of new ventures for the supportive environment they create through the provision of social, knowledge, and financial capital, all of which are well developed as necessary to the entrepreneurial process (Clough, Fang, Vissa, \& Wu, 2019; Spigel \& Harrison, 2018). Despite existing in the same region with presumably the same goals, the organizations providing entrepreneurial support have varying business models, underlying logics, and even end up working in sectors as either for-profit or non-profit (Roundy, 2017). Such diversity in business model and understanding within entrepreneurial ecosystems has been glossed over in current policy and scholarly discussions which assume cooperative behavior as a given; however, these differences can matter greatly in to how these organizations perceive one another and the interaction patterns that arise. Organizational interaction patterns refer to emergent and reinforcing configurations of relational exchange involving policies, resources, and communication between a focal organization and other organizations (Van de Ven, Walker, \& Liston, 1979). With a growing body of work on how diverse organizations across sectors interact (Hannah \& Eisenhardt, 2018; Mathias, Huyghe, Frid, \& Galloway, 2017) the regional nature of an entrepreneurial ecosystem brings additional challenges to cross-sector collaboration. While 
regional density and interaction between organization leaders presumably raises the chances of collaboration, geographic proximity does not ensure social proximity nor guarantee collaboration (Ben Letaifa \& Rabeau, 2013). The creation of entrepreneurial ecosystems is the subject of many government and policy directions, often in the form of millions of dollars in regional spending (Lerner, 2009) and yet scholarly work nor policymakers understand the challenges and opportunities that arise when organizations attempt to work together. Thus, we seek to answer the question "how do different interaction patterns emerge in entrepreneurial ecosystems" given the potential and incentives for breakdown in collaboration.

In this study, we investigate the Cambridge, UK entrepreneurial ecosystem to uncover the interaction patterns between entrepreneurial support organizations. We chose Cambridge as an extreme context given its reputation as a high-performing entrepreneurial cluster, geographic propinquity of organizations, and the diverse federated historical structure wherein a unique institutional environment of a collegial university and spinout organizations create a complex unstructured network of entrepreneurial support. We consider this a region where the tensions associated with a high diversity of organizations would exist: no overarching structure facilitates interaction or collaboration, which renders it useful for observing the tension that arises in this ecosystem. Our findings inductively surface a typology of four ecosystem interaction patterns, the ways in which organizations navigate reinforcing exchanges and partnerships which arise, both challenges and opportunities. We develop that these interaction patterns are rooted in the organizational leaders' perceptions of a regional ecosystem identity and the ways in which organizations' identity orientation combine with these perceptions. 
We make three contributions to existing theory. First, we develop a model that explains how interaction patterns between organizations emerge within entrepreneurial ecosystems. This primarily contributes to understanding the conditions under which entrepreneurial support organizations are likely and able to form productive and collaborative relationships across the entrepreneurial ecosystem despite the potential tensions associated with organizational differences. By contesting the assumption that organizations will inherently collaborate, we give insight for both policy and scholarly work on entrepreneurial ecosystems. Secondly, we extend research on collective identities by demonstrating how a strong perceived regional ecosystem identity can buffer associated organizations from pressures which might undermine the potential for cross-field and cross-sector interactions. Our third contribution extends the longstanding but loosely developed literature on organizational identity orientation. While prior research on organizational identity orientation has focused largely on stakeholder relations in the context of single industries, we extend this literature by illustrating how such orientations affect cross-sector interactions. Moreover, we illustrate how such orientations do not operate independently in shaping stakeholder interactions, but rather are equally informed by whether or not organizations perceive a strong regional collective identity.

\section{INTERACTION PATTERNS IN ECOSYSTEMS}

\section{Interaction Patterns Between Organizations}

Organizations do not exist in isolation and are rather part of a complex network of the various stakeholders, competitors, and collaborator organizations that are present around them. The interactions between organizations has been observed for decades (e.g. Schmidt \& Kochan, 1977) but is increasingly emphasizing the constituent reasons and benefits in doing so beyond a 
resource perspective: for organizations without direct competitive pressures between them, benefits of interactions can include knowledge and political spillovers (Hardy, Phillips, \& Lawrence, 2003) leading to innovation or new forms of organizing (Brass, Galaskiewicz, Greve, \& Tsai, 2004). Amongst competing organizations interactions occur and can lead to outcomes such as the formation of alliances, cooperatives, or joint initiatives as a mechanism to advance the interests of all parties (Hardy \& Phillips, 1998; Uzzi, 1997). Research on competition and collaboration by organizations has largely remained as two distinct streams with increasingly calls for an integrative approach to understand these at times conflicting, but often coexisting, interaction patterns (Hoffmann, Lavie, Reuer, \& Shipilov, 2018; Polenske, 2004).

A large body of research is focused on understanding competition and collaboration through identity considerations. Foundational work in strategic groups (Porac, Thomas, \& Baden-Fuller, 1989) and categorical constraints (Zuckerman, 1999) began a stream of research that would "focus on how the category structure of an industry, and the associated cognitive maps used by managers, affect competitive dynamics" (Vergne \& Wry, 2014: 62). Individual firms contending with identity challenges can be observed to undertake different strategies due to competitive pressures for organizations (Fisher, Kotha, \& Lahiri, 2016; Irwin, Lahneman, \& Parmigiani, 2018) or interactions with stakeholders (Tracey, Dalpiaz, \& Phillips, 2018; Tracey \& Phillips, 2016). Collective identity has been identified as a means to advance the status of the set of organizations cooperating and align them to a common mission for the purposes of attracting attention or legitimacy within a crowded space (Lounsbury \& Glynn, 2019; Mathias et al., 2017; Patvardhan, Gioia, \& Hamilton, 2015). 
In addition to looking at the identity challenges of a collective, a further stream of research looks at the ways in which organizations deal with interactions within the context of a single industry. Organizations may cooperate based on internal orientations towards interactions that are derivative of members understandings of who and what they are. In some cases it has been observed that different types of interactions emerge due to an identity orientation: an organization's self-conception of their interactions with other organizations and stakeholders (Brickson, 2005, 2007). Interactions with industry peers and stakeholders can be seen as individualistic, relativistic, or collectivistic, according to the underlying motivations and conceptions of the relationship by the focal firm. The "actions of collectivistic organizations are often motivated by a desire to serve collective interests and protect communal well-being" as part of an overarching socio-economic community, while "relational organizations often see relationships as an end in themselves... [creating] a desire to understand their stakeholders" (Gal, Blegind Jensen, \& Lyytinen, 2014). Organizational identity orientations provide a means to bridge research between organizational identity and the greater stakeholder audience outside of an industry.

\section{The Sustained Importance of Regions in Shaping Organizational Interaction Patterns}

The emergence of organizational interactions is not explained by the motivations or identities solely. Despite the increasingly globalized world organizations operate in and the seemingly diminished importance of regional dynamics scholarly work continues to recognize that regions are influential in the determination of interactions between organizations (Howard-Grenville, Metzger, \& Meyer, 2013; Marquis \& Battilana, 2009). A longstanding body of research has considered the role of regions in creating clusters of cooperation, or at the very 
least shared identity, both within- and across-sectors. Influences in creating industry clusters (Romanelli \& Khessina, 2005) focus on the need for strong regional identity to attract resources and develop collaborative mechanisms between organizations. Regional identity is an enabler of collaboration because of the affordances it provides to organizations as an overarching source of legitimacy, reputation, and shared ambition (Autio et al., 2018; Beck, Swaminathan, Wade, \& Wezel, 2019; Beebe, Haque, Jarvis, Kenney, \& Patton, 2013). The development of a collective identity follows a contented and contingent process undertaken by the members (Patvardhan et al., 2015) that enables and constrains legitimate interpretations of membership in the collective.

One basis for collaboration within a region is the advancement of the overall community. An organization aligned weakly to the regional identity may face reduced resource attraction or benefits come from the collective efforts of the community (Romanelli \& Khessina, 2005). Yet, despite the advancement of a regional identity, propinquity and presence in a region has been shown as potentially both a strong incentive for and a potential barrier to collaboration (Freeman \& Audia, 2006). Recent work has suggested that both the institutional framework and underlying logics of a region can influence cooperation but also incite tension (Balland, 2012; Ben Letaifa \& Rabeau, 2013). Embedded inside of a regional approach to interorganizational cooperation is the link between interpersonal and interorganizational relationships (Chetty \& Agndal, 2008; Hogg, Van Knippenberg, \& Rast, 2012). Geographic proximity places both organizations and their leaders within a network that becomes entangled, where interpersonal relationships provide both spillover benefits and reservation towards cooperation. Unlike a cross-sector or cross-field perspective on cooperation, the strong interpersonal effects are likely to lead towards more informal cooperative efforts based on 'who knows who'. Regions enable serendipitous and 
emergent forms of interorganizational cooperation in part due to these interpersonal relationships (Ahuja, Soda, \& Zaheer, 2012; Wry, Lounsbury, \& Glynn, 2011). The influence of regions on organizational interaction is one that cannot be ignored, particularly with respect to the interaction of organizations in a relationally-based field such as entrepreneurship.

\section{Organizational Interactions within Entrepreneurial Ecosystems}

Entrepreneurship has increasingly been understood as a process that relies on the presence and intervention of organizations providing sponsorship of new ventures and through their growth (Amezcua, Grimes, Bradley, \& Wiklund, 2013); accessing and mobilizing a variety of resources is paramount to the success of these ventures (Clough et al., 2019). As "systems of co-located elements where a variety of actors, functions, and institutions interact to support the creation and growth of new ventures" entrepreneurial ecosystems are the subject of recent work in understanding entrepreneurial activity (Thompson et al., 2018: 97). Unlike other types of ecosystems, entrepreneurial ecosystems are industry agnostic and focus on the creation and growth of new ventures (Thomas \& Autio, Forthcoming). Research on these regions has recognized the importance of having cultural and social attributes in addition to the material elements such as workspace, financial capital, or government tax incentives for example (Romanelli \& Khessina, 2005; Saxenian, 1994; Spigel, 2017). The configuration of these three attributes: material, social, and cultural, lead to the formation and reproduction of the unique identity of an entrepreneurial ecosystem.

Previous research has examined ecosystems across levels of analysis, considering specific components such as angel investors (Steier \& Greenwood, 2000) to the construction of a new ecosystem (Thompson et al., 2018). Common, however, is the presupposition that 
organizations in the ecosystem are intertwined and interacting in their support of new ventures. The organizations that exist in entrepreneurial ecosystems are often plentiful and diverse. Universities are often a central node within a region (Schaeffer \& Matt, 2016; Wright, Siegel, \& Mustar, 2017) and have long been posited as a key resource for entrepreneurial ecosystems (Saxenian, 1994). Incubators, often non-profit or government funded, provide access to resources to refine business models at the creative ideation stage (Dutt et al., 2016; Grimes, 2018) whereas accelerators are typically for-profit and aim to create a sheltered environment for new ventures to access increased resources and prepare for investment in a structured, cohort-based program (Cohen, Bingham, \& Hallen, 2018; Goswami, Mitchell, \& Bhagavatula, 2018; Pauwels, Clarysse, Wright, \& Van Hove, 2016). The sphere of funding resources span from informal investors and angel networks (Steier \& Greenwood, 2000) to institutional type investment from venture capital (Blevins \& Ragozzino, 2018). These are just some of the diverse institutions and organizations found within an entrepreneurial ecosystem crossing sectors, fields, and underlying logics. The co-location of these organizations in an entrepreneurial ecosystem lead to reinforced and recurring interaction between each other through formal and informal networks of leaders in particular, "actors with high levels of social capital who proactively build new connections between entrepreneurial actors, helping to improve firm formation and growth within region" (Spigel, 2017: 53).

Within the milieu of the entrepreneurial ecosystem, it becomes important to understand the conditions under which collaborative or competitive interactions emerge and the degree to which the ecosystem can then foster successful organizational sponsorship. In order to capture the benefits of the "potent policy tool to... create, deliver, and capture not only economic, but 
also societal, value" (Autio et al., 2018: 78), we require a more nuanced understanding of the factors that will advance an ecosystem towards one that is both diverse yet also productive in supporting entrepreneurs that is left unresolved by previous literature investigating interorganizational interactions and policy discussions.

\section{RESEARCH METHODOLOGY}

The Cambridge entrepreneurial ecosystem was selected given the presence of such organizations and its global recognition as a model of a successful entrepreneurial ecosystem (Kirk \& Cotton, 2012). Cambridge has over 50 organizations providing entrepreneurial support and has seen hundreds of millions of dollars invested in startup ventures. Cambridge is an ecosystem rich in history and success stories. Early startups including ARM, Solexa, and Autonomy have paved the way for an ecosystem that is tightly connected and is host to hundreds of entrepreneurial ventures across the cluster. The ecosystem is still dynamic and evolving: during the course of collecting data two new accelerators were launched and a multi-institution entrepreneurial initiative set in motion. This results in an ecosystem that is continually adapting to the presence of new understandings and perspectives of interorganizational collaboration and shared ambition.

Cambridge has a unique structure given the strong presence of the University of Cambridge and affiliated colleges which continue to play a central role in the city as its largest employer, land developer, and the connections which many startups, spinouts, and growth companies have. The federated historical structure of the region has crossover in economic development between the varying levels of government from municipal to parliament, with the university also contributing. This develops an ecosystem that faces great interest from 
policymakers and those who run entrepreneurial support organizations both at the university, for-profit, and non-profit community hubs.

The sample of organizations contacted was created through purposive sampling (Lincoln \& Guba, 1985; Silverman, 2014). The organizations represented a cross-sector sample of entrepreneurial support models including accelerators, incubators, co-working spaces, student organizations, and business networks. These organizations were selected to understand the effects of varying organizational identity and interactions that unfold in the Cambridge ecosystem. The sample did not however include traditional professional service providers (e.g. law offices, accountants) as they are not providers of organizational sponsorship, and therefore are outside the scope of interest of this study.

Insert Table 1 about here.

\section{Data Collection}

This project began in March 2019 and in total 15 interviews were conducted between March-December 2019. Initially, three initial face-to-face interviews were conducted with organizations from the Cambridge ecosystem after being contacted through cold emails. The interviews were conducted at the organization's office or a university office and ranged between 60-75 minutes. The further interviews were conducted after reviewing emergent themes and revising the interview approach. A semi-structured interview approach was selected to allow discussion of the main themes being investigated, but allowed for a more natural, conversational approach to the research. This also allowed for interviewees to share information about their 
organization that they felt was worth understanding, particularly with respect to how they viewed the Cambridge ecosystem, future directions, and challenges that they face as an organization. We made efforts to ensure reliability in the interviews in not providing any leading questions or sharing our own interpretation of the Cambridge ecosystem, nor views on specific organizations.

First-hand accounts of the Cambridge ecosystem were complemented with secondary data. For example, The Cambridge Phenomenon: 50 Years of Innovation and Enterprise (Kirk \& Cotton, 2012) provides a historical narrative of the founding and growth of the Cambridge technology cluster and insight into some of the collaborative work seen in the ecosystem. Other specialist reports and annual reports from ecosystem organizations available in the public domain were reviewed. These were used to sensitize the researchers to the various stakeholders, activities, and connections that exist in the region.

\section{Data Analysis}

This research followed an inductive approach. Theoretical developments are emergent from through the data collection and analysis and this approach was chosen based on the limited amount of research in the domain of entrepreneurial ecosystems. Inductive approaches focus on "an iterative process of gathering raw data, producing progressively better-defined and grounded higher-order concepts through constant comparison and mind-expanding techniques, and creating underlying theoretical arguments that connect constructs" (Eisenhardt, Graebner, \& Sonenshein, 2016: 1114). While building theoretical insights, the researcher develops the emergent model with both a strong insight from participants and the inclusion of relevant theoretical and contextual background. 
We followed a five-stage process in analyzing the data; although described sequentially, this involved moving between the data, emergent frameworks, and extant literature throughout the process (Locke, 2003).

Stage 1: We transcribed the interview recordings gathered during this research in their entirety, which were compiled in Atlas.TI, a computer aided qualitative data analysis tool. These were transcribed within a short time after the interviews to allow field notes taken during and post-interviews to be connected to the transcripts. Notes compiled from secondary materials were incorporated in this stage to develop a comprehensive understanding of the Cambridge ecosystem and connections between organizations.

Stage 2: We followed the constant comparison method, which began in the first stage with open coding (Corbin \& Strauss, 1990; Van Maanen, 1979). Coding was conducted at the sentence and paragraph level, where descriptive codes were developed highlighting overall understanding of identity in the entrepreneurial ecosystem, identification with category labels, and interaction patterns between organizations in the Cambridge cluster. Representative codes included "shared mission is present and adhered to" or "adapted to the local ecosystem".

Data analysis was ongoing throughout the research process with transcription and coding taking place in between interviews, the results and findings of which informed areas to probe and discuss in subsequent interviews. This led to the adaptation between interview guides after the first three interviews, focusing on the role of the wider ecosystem in organizational identity.

Stage 3: Afters several interviews were conducted, first-order open codes were used to develop second-order themes. Key categories emerged and were highlighted across the interviews as salient questions organizations faced in their interactions in the Cambridge 
ecosystem. Varying perceptions of a shared mission were described and highlighted as a point of contention between various partners. Organizations were found to engage in varying practices of category buffering: the importance of recognition and legitimacy outside the Cambridge ecosystem was highlighted. Finally, the collaboration and interactions that organizations had with others in the ecosystem were noted to vary across those interviewed.

Stage 4: In this stage, we worked through a process of selective coding, which aims to identify and build relationships between the categories and themes. Based on the second-order themes, we created aggregate dimensions that were used in constructing a theoretical model (Figure 1) that relates the perception of collective identity, category buffering, and interaction patterns. This identified four types of organizational interaction patterns that emerged in the ecosystem (Figure 2). These were drawn from both extant literature and the framework of interaction patterns that was developed. These were recursively validated to ensure consistency, one measure of trustworthiness of findings in qualitative research (Seale, 1999). The dimensions and emergent interaction patterns were further developed as subsequent interviews were conducted to refine, validate, and build the model.

Stage 5: To further provide trustworthiness to the theoretical findings, we engaged in a form of member-checking (Nag, Corley, \& Gioia, 2007). In this case, the results were shared with an entrepreneurship advocate known to the lead researcher from outside the Cambridge ecosystem to understand the transferability of the findings. She provided added critical reflection to the model and highlighted differences that may exist in the Cambridge ecosystem not seen elsewhere. 


\section{RESEARCH FINDINGS}

\section{Emergence of Ecosystem Interaction Patterns}

We first surface a theoretical model that demonstrates the emergence of interaction patterns between organizations in an entrepreneurial ecosystem. This model introduces new concepts in understanding entrepreneurial ecosystems as regions of organizational interactions. We focus on two key mechanisms in the unfolding of these interaction patterns, referred to as perceived ecosystem identity and ecosystem organizational identity orientation.

Insert Figure 1 about here.

Entrepreneurial ecosystems can be examined as both components or as an overarching collective. Defining entrepreneurial ecosystems as regions and an organized set of actors allows us to consider that the ecosystem has a regional identity. This is a form of collective identity shaped by interorganizational collaboration (Hardy et al., 2003; Patvardhan et al., 2015). Not all organizations articulate that a shared or regional identity exists in the Cambridge region; even amongst those that do believe a regional identity exists, the interpretations of such are varied. Thus, we introduce the concept of perceived ecosystem identity. This is a collective identity and is comprised of the shared understanding of norms, discourse, and shared mission between those in a regional entrepreneurial ecosystem. Previous literature has articulated this through higher-order shared missions between organizations and social attributes within an ecosystem (Patvardhan et al., 2015; Spigel, 2017). The perceptions of such an identity will vary between entrepreneurial support organization leaders, some of whom believe a collective identity exists 
and affects the way they operate their organization to others who do not believe it exists and thus has no impact on their interactions. We find that these varying perceptions by leaders influence their interactions with other organizations.

Alpha: I think Cambridge has a nice shared mission actually. And I think a lot of people would nod and agree.

Chi: I would say there is definitely not a common shared mission. I mean there is at a high level, everybody that comes to Cambridge is pretty loyal to the ecosystem... but that's probably it for a shared mission.

The perceived ecosystem identity is important for understanding interaction patterns between organizations because of the affordance it provides. Our model surfaces that organizational category buffering is afforded by a stronger sense of collective identity by an organization in the ecosystem. Traditional understanding of the categorical imperative (Zuckerman, 1999) articulates that organizations will largely conform to the category they belong to. In an entrepreneurial ecosystem, this would presuppose that we would see a set of organizations that very clearly identify with a higher market category such as 'accelerator' or 'incubator' and match their practices and organizational identity with those of prototypes in their category (often being a North American model such as TechStars, YCombinator, etc). Instead, leaders who find that they resonate with an ecosystem identity can lead their organization to break away from the perceived categorical imperative, as their sense of a shared mission in the ecosystem affords a form of legitimacy that lowers the expectations of conformity. Leaders which believe the ecosystem has a strong regional identity are more willing to deviate from their own category, given the legitimacy the greater ecosystem provides them. This is found in the 
Cambridge ecosystem with organizations drifting from the organizational prototype through differentiated programming, business model, or funding relationships. Entrepreneurial support organization leaders with a less pronounced perception of an ecosystem identity are not afforded the same buffering of the categorical imperative and are more likely to follow their respective organizational category.

Organizational identity orientation has been largely considered through the interaction between organizations and their stakeholders; in an entrepreneurial ecosystem, these stakeholders are bounded from a regional perspective. We therefore consider an organization's ecosystem organizational identity orientation: this is the view of an organization's interactions with a second organization, vis-à-vis the dyad partner. Their attention or inattention to partnerships and stakeholders define an organizational identity orientation. Each organization has a unique, yet consistent orientation, and thus leads us to understanding the interactions of the various accelerators, incubators, coworking spaces, capital providers, and student organizations that make up the Cambridge ecosystem.

While three distinct identity orientations have been modelled (individualistic, relativistic, and collectivistic), these are not all equally legitimate in an entrepreneurial ecosystem. We find that organizations in the Cambridge entrepreneurial ecosystem have one of two identity orientations, either relativistic or collectivistic. This was highlighted with regards to organizations that have come and subsequently left the ecosystem: those who failed to engage with the ecosystem are looked upon poorly and do not receive the support or all-important referrals from other ecosystem organizations. This is important as it highlights how the ecosystem collectively sanctions organizations who do not participate or interact broadly. 
Interaction patterns are observed behaviors that occur between individuals or organizations. We propose four distinct interaction patterns and propose a model that places organizational identity orientation as an effect linking organization's identity and ability to engage in category buffering (Figure 2). We develop that this determines whether organizations primarily partner with other ecosystem organizations, build ecosystem capacity, participate when it is beneficial, or bridge their identity with the ecosystem missions.

The intersection of these two theoretical constructs, both organizational identity orientation and perceived ecosystem identity, develops a $2 \times 2$ framework and typology of entrepreneurial support organizations found in the Cambridge ecosystem (Figure 2). Organizations are characterized through their interaction patterns and perceptions of activity between other organizations in the ecosystem, and their own identification with greater categorical prototypes and models. These are viewed as mutually exclusive and exhaustive due to the theoretical underpinnings of the typology.

\section{Insert Figure 2 about here}

\section{Orientating to the ecosystem}

Organizations in the Cambridge ecosystem have clear understandings of their own aims and the types of relationships required to achieve them. The outward projection of these aims and the partnerships that are generated create a frame of organizational identity orientation that can be articulated by both the organization itself and other members of the ecosystem. Gamma is "trying to catalyze the activity inside the space and the people that come here come from many 
different places." Their service offer as a shared workspace further allows them to provide opportunities and serendipitous connections to emerge for their customers. To do so requires a collaborative view of the ecosystem to enable and advance these goals. They frame the role of an organization to be a collaborator in advancing the ecosystem. This is highlighted both by the way the organizations discuss their own activities and further reinforced when other organizations share their experience working with them. Alpha views their programme as an open-source approach to entrepreneurial sponsorship:

Alpha: I really am trying to blow this open, this will sort of be the open source software of incubator programs going forward. And we should not be attributed to their success I should think, but hopefully by being the coordinator of all of this and making those introductions it should serve the ventures better and ultimately what we really want is to have those businesses solving those challenges. That's the most important thing. It's not about who has a badge on it.

This ambition is reinforced as a legitimate claim by other organizations in the ecosystem. Atom discusses that Alpha is particularly known for engaging others "we'll do joint events with [Alpha]... we're at the early stages of those joint ventures, but the plan is to do more joint stuff." Builder organizations like Alpha and Beta are both working towards enhancing the overall availability of services and connections across the ecosystem.

Beta: We're kind of different. We get asked to replicate what we do around the world in places. There aren't really many organizations like [Beta]... what we do is get the businesses and the academic institutes to share the things that are not differentiating for each other in Cambridge but can differentiate us as a whole. 
The role of Beta is centered around their collectivistic mindset - the whole is stronger than the parts - and collaborative programs can only advance the individual organizations. Beta fill specific needs and bring together the greater community. This makes them incredibly valuable here, but it is noted how "others may fail to see the value" elsewhere. Their value is adapted based on the collective needs of the Cambridge ecosystem uniquely. Builder organizations think in terms of the benefits to the greater ecosystem aiding their own success: the health of the ecosystem is the responsibility of everyone including themselves. This is highlighted through work that breaks down barriers between organizations, positioning them as a hub of social capital rather than a spoke in the complex network.

Alpha: Where I think the community works really well and has brought us together better than anything else in my opinion is [Xi]'s effort in pulling together the UEN University Enterprise Network... I think it was about 36 of us who came around the table the last time...that's an opportunity... that you've got those connections and it all feeds in.

Both Alpha and Xi demonstrate this type of community building work and are referenced by other organizations in the ecosystem as strong collaborators because of their willingness to work with everyone. They are aware of their position and strengths in this area and refer to it as an advantage in the delivery of their programming, "I think [Alpha] is quite naturally positioned to be a partner to others" and "it's very much the community aspect that makes [Xi] work well" being examples of positioning. Beta refers to themselves as a "super connector" in the ecosystem. They use this to attract potential partners for program development and as a unique position to attract entrepreneurs to join them, focusing on the sense of connection to the greater 
community that they can provide. Xi was instrumental in the successful creation of another organization in the ecosystem. Reflecting, they noted how they "think a big thing was that we were tapping into an undercurrent in the community. And we weren't creating any demand that wasn't there, just unlocking it. And we used a lot of social capital to make that work." This did not provide particular benefit to Xi but was done to grow the collective capacity of the ecosystem.

Other organizations interact through dyadic partnerships that are formed based on the needs of each organization. These relationships represent particular transactions: connecting mentors to participants of their program or working with other ecosystem members to cross-populate programming such as events or future programs.

Sigma: This is a platform where we try to bring those actors together, and I guess during the talks we provide like space and time for people to meet and discuss a topic... Through the startup accelerator as well, people that have an invention or a product they might want to develop, they need a mentor or advice... we help in trying to make those connections.

The focus is on fulfilling a need in one or both partner organizations with less of a view on connecting multiple stakeholders together. The partnerships established by these organizations are driven by pragmatism to fill specific gaps identified in the ecosystem where the brand and know-how can contribute to greater success. The leaders of these organizations describe that a social contract exists in working together, but a shared purpose is not driving these interactions. The relationships formed are about creating additional capacity in the ecosystem. Chi and Delta address very salient gaps in the ecosystem, but view their role as 
closing it rather than changing interactions or perspectives amongst entrepreneurs and other organizations.

Delta: We are here to develop and boost a genomics ecosystem... we know the area we need to focus on. If we can play a role in being narrow and going in depth, we will find our place in the ecosystem.

Chi: We're not trying to change people's behavior, which is if you need a million pounds don't go to X, come to [Chi], that would be a way of manipulating the cluster a little bit to our own benefit. We're trying to go a slightly different way: early stage businesses with seed investments, keep doing what you're doing, right. We're just addressing the bottleneck to grow big right now that exists.

Chi and Delta both emphasize the need to have the right partners along the way to make their activities possible. Chi relies on their stakeholders to grow and achieve their own goals, partnering and building relationships in a one-to-one manner to advance outcomes and create capacity for future deals and venture growth in the region. This contrasts with the collectivistic perspective which relies on capacity creation through collective, one-to-many relationships.

\section{Perceptions lead to buffering}

Entrepreneurial ecosystems are made up of a broad array of different support services and organizations. We find that there is a vast difference in interpretation and adherence to categorical prototypes for these organizations, leading further to the diverse organizations and interaction patterns across the ecosystem.

Iota: We never have formally done any kind of back to back analysis, you know some kind of comparison chart, spreadsheet, something like that. We tick these boxes, they 
don't. Obviously, we are aware of the other programs, we are very much open to adopting best practice... [but] we think [our program] is pretty well optimized for the ventures that we want to work with.

Organizations such as Iota are less concerned about their outward appearance as aligning to a category in part due to their strong position in the ecosystem and belief in a shared identity across the region. The links between having a broad internal identity and strong perception of the "Our role is to be there at the beginning and then pass it onto the market... if you look at our mission which is to create societal impact, that's pretty big." Other organizations are competing on a global scale or are following models found elsewhere. Chi identifies clearly with a category and practices that match with that. Rather than using the strength of the ecosystem to adapt or align towards other goals, they remain outwardly identifiable as a clear representation of the category.

Chi: I know that [this] is a bit boring and superficial, but the whole reason we are here is to give financial return back to our investors. Of course we are interested in social impact and making Cambridge a better place, but our primary mission is to seek great returns... you're either a top quartile fund or you're not essentially.

Comparisons to other organizations come by way of how they align to best practices and benchmarking. For some, that is about returns or types of companies supported. For others, it is about the types of services or ethos they carry. "We see ourselves very much as an incubator... it's the stuff they don't know... it's about sitting down and introducing them to mentors and that kind of stuff, who can help them take their business from where they are today to be in 12 to 24 months' time." Clear adherence to a category gives an organization legitimacy beyond the local 
ecosystem. The coherency of an ecosystem identity is less perceived and thus requires the clear adherence.

$P i$ : In the true sense of the word, there are not many incubators around here. And I think it is because that business model doesn't really work. Most of them are accelerators, and most of them run a sort of rigid twelve week course if you like... it's very much the American way... That doesn't work for everyone, so we try and tailor things to be more bespoke...

Pi has a business-centered proposition, in that the incubator has to cover costs and pay for itself. This is used to contrast themselves from other organizations in the ecosystem that are grant or publicly funded. This requires outward alignment to similar organizations, especially to ensure their business's viability. They navigate the tension between 'sameness' and being bespoke by relying on the needs and identity of the ecosystem.

\section{Connecting buffers and orientations}

Across the Cambridge ecosystem we observe nuanced interaction patterns between the varying organizations providing sponsorship. This is at a level that extends beyond purely dyadic partnership or collectivistic actions as a hub. Rather, it develops the role that organizations wish to take in the ecosystem and the patterns which are demonstrated to achieve that end.

Partners in the ecosystem are driven to achieve individual organizational goals while adapting to the local ecosystem. These organizations are "bespoke" or constructed over time through identified gaps in the ecosystem. They develop specific connections to other organizations based on cross-promotion, shared expertise, or enhancing their role in the ecosystem. Rather than focusing on developing themselves according to a prototypical model, 
they adapt and promote the ecosystem through the curated partnerships engaged in. Builder organizations think in terms of the benefits to the greater ecosystem aiding their own success: the health of the ecosystem is the responsibility of everyone including themselves. They facilitate the coming together of various stakeholders with programming and initiatives that focus on the growth of ecosystem capacity. This is highlighted through work that breaks down barriers between organizations, positioning them as a hub of social capital rather than a spoke in the complex network. Participant organizations follow models that exist outside the local region and actively benchmark themselves against their nearest competitors and models from abroad. The partnerships established by these organization are undertaken to fill specific gaps identified in the ecosystem where the brand and know-how can contribute to greater success. Bridgers are organizations that act primarily as hubs for individuals and community members from across the ecosystem yet connect to a clear model and organizational ambition. These organizations have a purpose of bringing people together and connecting them across the community, filling a variety of roles from space to referrals.

This collection of interaction patterns promotes a diverse ecosystem from a perspective distinct from a variety of organizational sponsorship forms. Rather, the ecosystem is built on the basis that organizations will play a role in creating, defining, and maintaining the relationships needed to support new ventures. To achieve the aim of creating a munificent environment for ventures to grow in requires partnerships of various forms and organizations taking on roles to promote interactions. Nearly all organizations echoed comments such as "we need [to, and] we can work more closely with the other incubators in Cambridge" and the need to reinforce 
relationships that can otherwise "wane over time". Ultimately, no organization fills the whole puzzle and the interactions between them are what enable the ecosystem to thrive:

Xi: We wouldn't be where we are without the larger ecosystem. We are a very small part of a wider community. And we try to do a very narrow part of what our founders need. We are literally a team of four people. We have 220 members at the moment and 120 companies. There is no way that we could do what they need but hopefully we provide a platform for the rest of the community to get to them.

\section{DISCUSSION}

Beginning with the tension of how diverse organizations interact in entrepreneurial ecosystems, we surface a set of salient identity constructs that can uncover the ways in which organizations adapt, collaborate, and foster the development of the ecosystem. In the tradition of previous work (Howard-Grenville et al., 2013; Romanelli \& Khessina, 2005) our findings suggest that a regional identity plays a central role in the collaborative nature of organizations, such that leaders in the ecosystem can identify opportunities to adapt their programming and organizational category to build capacity and foster interorganizational collaboration. Our model differs from previous work on entrepreneurial ecosystems in that we explicitly attend to the diversity of organizations with respect to their role-illustrated by the interaction patterns we raise-in the ecosystem rather than diversity of service offerings. Here elaborate on our contributions and illustrate how our model builds understanding in other collaborative environments. 


\section{Emergence of Ecosystem Interaction Patterns}

Review of the previous literature surrounding entrepreneurial ecosystems has developed little in understanding how the diverse set of organizations found in these regions come together and interact. This paper has further developed the conceptual and theoretical understandings of entrepreneurial ecosystems and answers the research question of how different interaction patterns emerge amongst and between organizations. Previous understandings of ecosystems consider how various attributes such as the material, cultural, and social properties of a region and relationships between them construct the outcomes and cooperation found there (Spigel, 2017; Thompson et al., 2018). However, this does not resolve the question of how organizations with different underlying logics, whether they be for-profit, non-profit, government-mandated, or otherwise come together or pull apart, given all of their differences.

To resolve this tension, we introduce the construct of perceived ecosystem identity. Understanding how different organizations work together requires an understanding of what binds or causes interaction to begin with. When considering entrepreneurial ecosystems, resource dependence or a traditional supplier-purchaser arrangement does not exist, in contrast to traditional business or innovation ecosystems. The resources that entrepreneurial support organizations seek are high-quality entrepreneurs and new ventures they can support. This drives the promotion of a perceived ecosystem identity, as each organization realizes that this cannot be achieved solely without any greater collective. Developing a shared identity is not necessarily uncontested, however. Other forms of ecosystems such as innovation clusters or R\&D alliances are bound together by market-driven goals or formed to solve particular challenges facing firms. 
Organizations in entrepreneurial ecosystems require a binding force driving every organization towards the same goal.

While many individual interactions in the ecosystem occur, few efforts pull together each and every organization to allow for traditional collective identity to be crafted through storytelling or sensemaking (Wry et al., 2011). The model introduced in this paper begins to uncover some of the mechanisms answering how a collective identity is used by organizations, even when the identity is not salient among the entire audience. Even organizations who do not ascribe to the idea of an ecosystem identity identify that they must follow a set of common values and patterns of interaction. The degree of centrality, or even clarity, is thus less important in a regional context as it is shown to still produce a binding quality for these organizations. The development of collective level identity is an area of research called from by identity scholars (Gioia, Patvardhan, Hamilton, \& Corley, 2013) and this paper begins to articulate the value of such an identity in cooperative organizational relationships.

We build on previous research of entrepreneurial ecosystems and cooperation which emphasizes the need for interaction and collaboration to thrive (Thompson, Purdy, and Ventresca, 2018). Our research uncovers a unique set of interaction patterns that demonstrate the ways in which organizations utilize policies, resources, and communication to reinforce relationships across the ecosystem. While intuitively entrepreneurial ecosystem design may focus on highlighting and celebrating organizations who are 'super connectors' or hubs in the network, it is important to recognize that dyadic and collectivistic relationships go hand-in-hand in constructing and maintaining a healthy ecosystem. To that end, are there consequences from having an abundance of particular interaction patterns and a lack of others? Can organizations be 
incentivized to interact in any beneficial manner? The answers to these questions may lead policymakers to design interventions that encourage productive interaction patterns within entrepreneurial ecosystem to emerge.

\section{Challenging the Categorical Imperative}

The categorical imperative is a core in our understanding of market categories and how organizations shape their identity (Zuckerman, 1999). However, we understand that organizations are constantly balancing a degree of conformity while also standing out (Durand \& Paolella, 2013). This is particularly relevant in entrepreneurial ecosystems as we have found that organizations make claims of who they are and the category they belong to, yet find that often they are quite far from the category they claim membership in. To understand such patterns, we introduce the link between ecosystem identity and category buffering.

Category buffering is the process in which organizations take position themselves through identity claims and their programming or service offer to a particular category, however only to the extent required to achieve legitimacy in their operating environment. This distances them from the category they belong to. The degree of category buffering available to an organization has previously been defined solely by the categorical imperative. Instead, we find evidence that despite meaningful categories existing in entrepreneurial ecosystems, those such as business acceleration and venture capital, there are cases where the categorical imperative is weakened. This extends the categorical imperative in such that it can be moderated by other influences, in this case, perceived ecosystem identity. Previous work has suggested that "categorical claims may be predicated on a certain form of cultural resonance, in that organizations may seek to appropriate categorical memberships that align them well with their 
social, environment, or technical environments" (Glynn \& Navis, 2013: 1128). An understanding and perception of an ecosystem identity allows for organizations to expand or differentiate, knowing that such a collective identity offers a "stamp of approval" (Lerner, 2009) to organizations operating inside of it. This is articulated by members of the ecosystem through expressions such as how "this would only work in Cambridge" and their format "would not fit in elsewhere". Cambridge as a brand, and the perception of a shared ecosystem identity encourages and affords a weakened categorical imperative.

This builds on the concept of identity buffering introduced by Syakhroza et al. (2018), where organizations were shown to employ code-violations from their respective categories in their introduction of varying banking products. This work developed how the categorical imperative could be weakened and strengthened through varying cultural perceptions of an organization. Through the ability to employ an ecosystem identity, organizations in an entrepreneurial ecosystem can adapt to their local environment. This research adds to emerging literature that conceives of the categorical imperative as not an absolute construct, instead one that is flexible by cultural and social perceptions (Durand \& Paolella, 2013; Glynn \& Navis, 2013; Syakhroza et al., 2018). Category and identity buffering are demonstrated as an activity that allows for a diverse set of organizations to adapt beyond their organizational category to fulfill the needs of the entrepreneurial ecosystem and demonstrate value to stakeholders without suffering from illegitimacy or sanction. Does the presence of certain organizations in an ecosystem promote or weaken the ability for organizations to engage in category buffering? What preconditions are necessary for organizations to wish to adapt beyond their category, in addition to a strong sense of regional identity? 


\section{Contextualizing Organizational Identity Orientations}

Organizational identity orientation was introduced as an extension of literature surrounding individual identity orientations which began to theorize the interactions and identification of agents within organizations (Albert, Ashforth, \& Dutton, 2000; Brickson, 2000; Flynn, 2005).Since being raised by Brickson (2005, 2007), further work on identity orientation has been limited. Some studies have utilized the perspective to refine understanding of stakeholder relations and corporate social responsibility (Gal et al., 2014; Wickert, Vaccaro, \& Cornelissen, 2017). We expand organizational identity orientation beyond the single-industry context that it has previously been examined within. By examining the interactions between organizations within different sectors and fields, we develop a new way of seeing relationships between organizations and their stakeholders, in this case being ecosystem partners and competitors. Organizational identity orientations are an important construct in the interactions between diverse organizations, because of the focus on the perceptions that other stakeholders have of the organization.

This paper contextualizes organizational identity orientation through the model of interaction patterns in entrepreneurial ecosystems. We develop this as an important factor in determining how organizations utilize a shared regional ecosystem identity to adapt their interactions beyond solely individual organization goals. While this research uncovered organizations that orient in a relativistic and collectivistic manner, further study of the interactions that unfold when additional individualistic organizations are introduced would yield greater understanding of ecosystems. In a tightly bound ecosystem such as Cambridge, there is an emphasis on doing good and doing right by others; in an ecosystem less tightly bound through 
collective identity, sanctioning of improper orientations is less likely to occur. This expands our understanding of how interactions unfold, driven in part by the expectations of others and how tightly bound a region of diverse organizations is. Difficulties arising in partnerships and collaborative efforts might be examined to see if conflicting orientations lead to adverse outcomes. Are certain dyads of identity orientations incompatible?

\section{Implications for Building Healthy Ecosystems}

First, we find that there are two key components to understanding the diversity of these organizations: their perceptions of an ecosystem regional identity, and their ecosystem organizational identity orientation. This is influenced by the category buffering either afforded to them or not, which develops the diverse set of organizations found in a region. We develop a new perspective on how category and identity buffering can take place, through the development and perception of an ecosystem identity. Rather than developing that such an identity must exist and be agreed upon, this develops how merely a perception of such an identity existing is sufficient to allow organizations to adapt without suffering from illegitimacy costs in a local entrepreneurial ecosystem.

Second, we begin to resolve calls for how to understand collaboration with entrepreneurial ecosystems. Instead of focusing on the degree to which organizations compete or collaborate, we develop that organizations interact in four distinct patterns, as builders, bridgers, partners, or participants. This brings important practical understanding of how entrepreneurial ecosystems may unfold. Entrepreneurial ecosystems are best served through developing a regional identity that affords flexibility in organizational identification: well-developed 
entrepreneurial ecosystems are also known for their strong regional brand and identity which allows for organizations to adapt within them providing increased resources for entrepreneurs.

As policymakers and organizations leading the development of these regions work towards their growth, they should focus on building shared understanding and perception of cooperation, while also recognizing that unique ways of interacting will unfold. The typology of interaction patterns develops that an expectation of all organizations providing equal cooperation and partnership into the ecosystem is unrealistic, but the varying ways in which organizations participate can be leveraged.

\section{Limitations and Future Research}

The insights gained through this research are based on the investigation of a 'world leading' entrepreneurial ecosystem and innovation hub, a specific choice made to illuminate the tensions found between diverse organizations. Moreover, the region is quite small and well-connected. This choice allowed for us to observe a critical number of organizations as cases to inductively surface our model involving the perceptions of leaders and corresponding organizational identity constructs. However, in our selection of this case, it also provides an opportunity for further investigation of the emergence of interaction patterns in an ecosystem that is less connected or nascent rather than established. While Cambridge provides a context when such a perception is salient amongst many organizations, this may not hold in other ecosystems. Similarly, while perception of regional identity is one factor that influences the ways in which organizations interact, other factors such as community culture may play an important role in the collaborative efforts within an ecosystem. Scholars may look to understand 
communities where a strong cultural heritage shapes the entrepreneurial process, for example, in Indigenous communities (Lee \& Eversole, 2019).

Secondly, the influencing factors of organizational identity orientation remain undertheorized. While previous studies have suggested that the types of stakeholders that organizations interact with are the key factor influencing an organization's identity orientation, when considering this concept through in a regional ecosystem other factors appear as though they may be salient. Exogenous factors such as history in the ecosystem, age, experience, and past performance may be important factors in how an organization orients itself to the ecosystem. This is both a limitation in understanding the current model and also an opportunity for further research of this construct. Moreover, what is the outcome of collaboration when different organizational identity orientations intersect? When different understandings of collaboration are brought together, this may lead to tensions or misaligned expectations, however the extent to which this is of importance is left unresolved. Future studies could illuminate this interaction, further refining our understanding of how organizations collaborate in ecosystems.

\section{REFERENCES}

Ahuja, G., Soda, G., \& Zaheer, A. 2012. The Genesis and Dynamics of Organizational Networks. Organization Science, 23(2): 434-448.

Albert, S., Ashforth, B. E., \& Dutton, J. E. 2000. Organizational Identity and Identification: Charting New Waters and Building New Bridges. Academy of Management Review, 25(1): 13-17.

Autio, E., Nambisan, S., Thomas, L. D. W., \& Wright, M. 2018. Digital affordances, spatial affordances, and the genesis of entrepreneurial ecosystems. Strategic Entrepreneurship Journal, 12(1): 72-95.

Balland, P.-A. 2012. Proximity and the Evolution of Collaboration Networks: Evidence from Research and Development Projects within the Global Navigation Satellite System (GNSS) Industry. Regional Studies, 46(6): 741-756.

Beck, N., Swaminathan, A., Wade, J. B., \& Wezel, F. C. 2019. Industry Clusters and Organizational Prototypes: Evidence From the Franconian Brewing Industry. Journal of Management, 45(7): 2978-3008. 
Beebe, C., Haque, F., Jarvis, C., Kenney, M., \& Patton, D. 2013. Identity creation and cluster construction: The case of the Paso Robles wine region. Journal of Economic Geography, 13(5): 711-740.

Ben Letaifa, S., \& Rabeau, Y. 2013. Too close to collaborate? How geographic proximity could impede entrepreneurship and innovation. Journal of Business Research, 66(10): 2071-2078.

Brass, D. J., Galaskiewicz, J., Greve, H. R., \& Tsai, W. 2004. Taking Stock of Networks and Organizations: A Multilevel Perspective. Academy of Management Journal, 47(6): 795-817.

Brickson, S. 2000. The Impact of Identity Orientation on Individual and Organizational Outcomes in Demographically Diverse Settings. Academy of Management Review, 25(1): 82-101.

Brickson, S. L. 2005. Organizational Identity Orientation: Forging a Link between Organizational Identity and Organizations' Relations with Stakeholders. Administrative Science Quarterly, 50(4): 576-609.

Brickson, S. L. 2007. Organizational Identity Orientation: The Genesis of the Role of the Firm and Distinct Forms of Social Value. Academy of Management Review, 32(3): 864-888.

Chetty, S., \& Agndal, H. 2008. Role of Inter-organizational Networks and Interpersonal Networks in an Industrial District. Regional Studies, 42(2): 175-187.

Clough, D. R., Fang, T. P., Vissa, B., \& Wu, A. 2019. Turning Lead into Gold: How Do Entrepreneurs Mobilize Resources to Exploit Opportunities? Academy of Management Annals, 13(1): 240-271.

Cohen, S. L., Bingham, C. B., \& Hallen, B. L. 2018. The Role of Accelerator Designs in Mitigating Bounded Rationality in New Ventures. Administrative Science Quarterly.

Corbin, J., \& Strauss, A. 1990. Grounded Theory Research: Procedures, Canons, and Evaluative Criteria. Qualitative Sociology, 13(1): 1-21.

Durand, R., \& Paolella, L. 2013. Category Stretching: Reorienting Research on Categories in Strategy, Entrepreneurship, and Organization Theory: Reorienting Research on Categories. Journal of Management Studies, 50(6): 1100-1123.

Dutt, N., Hawn, O., Vidal, E., Chatterji, A., McGahan, A., et al. 2016. How Open System Intermediaries Address Institutional Failures: The Case of Business Incubators in Emerging-Market Countries. Academy of Management Journal, 59(3): 818-840.

Eisenhardt, K. M., Graebner, M. E., \& Sonenshein, S. 2016. Grand Challenges and Inductive Methods: Rigor Without Rigor Mortis. Academy of Management Journal, 59(4): 1113-1123.

Fisher, G., Kotha, S., \& Lahiri, A. 2016. Changing with the Times: An Integrated View of Identity, Legitimacy, and New Venture Life Cycles. Academy of Management Review, 41(3): 383-409.

Flynn, F. J. 2005. Identity Orientations and Forms of Social Exchange in Organizations. Academy of Management Review, 30(4): 737-750.

Freeman, J. H., \& Audia, P. G. 2006. Community Ecology and the Sociology of Organizations. Annual Review of Sociology, 32(1): 145-169.

Gal, U., Blegind Jensen, T., \& Lyytinen, K. 2014. Identity Orientation, Social Exchange, and Information Technology Use in Interorganizational Collaborations. Organization Science, 25(5): 1372-1390. 
Gioia, D. A., Patvardhan, S. D., Hamilton, A. L., \& Corley, K. G. 2013. Organizational Identity Formation and Change. Academy of Management Annals, 7(1): 123-193.

Glynn, M. A., \& Navis, C. 2013. Categories, Identities, and Cultural Classification: Moving Beyond a Model of Categorical Constraint: Categories, Identities, and Cultural Classification. Journal of Management Studies, 50(6): 1124-1137.

Goswami, K., Mitchell, J. R., \& Bhagavatula, S. 2018. Accelerator expertise: Understanding the intermediary role of accelerators in the development of the Bangalore entrepreneurial ecosystem. Strategic Entrepreneurship Journal, 12(1): 117-150.

Grimes, M. G. 2018. The Pivot: How Founders Respond to Feedback through Idea and Identity Work. Academy of Management Journal, 61(5): 1692-1717.

Hannah, D. P., \& Eisenhardt, K. M. 2018. How firms navigate cooperation and competition in nascent ecosystems. Strategic Management Journal, 39(12): 3163-3192.

Hardy, C., \& Phillips, N. 1998. Strategies of Engagement: Lessons from the Critical Examination of Collaboration and Conflict in an Interorganizational Domain. Organization Science, 9(2): 217-230.

Hardy, C., Phillips, N., \& Lawrence, T. B. 2003. Resources, Knowledge and Influence: The Organizational Effects of Interorganizational Collaboration*. Journal of Management Studies, 40(2): 321-347.

Hoffmann, W., Lavie, D., Reuer, J. J., \& Shipilov, A. 2018. The interplay of competition and cooperation. Strategic Management Journal, 39(12): 3033-3052.

Hogg, M. A., Van Knippenberg, D., \& Rast, D. E. 2012. Intergroup Leadership in Organizations: Leading Across Group and Organizational Boundaries. Academy of Management Review, 37(2): 232-255.

Howard-Grenville, J., Metzger, M. L., \& Meyer, A. D. 2013. Rekindling the Flame: Processes of Identity Resurrection. Academy of Management Journal, 56(1): 113-136.

Irwin, J., Lahneman, B., \& Parmigiani, A. 2018. Nested identities as cognitive drivers of strategy. Strategic Management Journal, 39(2): 269-294.

Lee, E., \& Eversole, R. 2019. Rethinking the regions: Indigenous peoples and regional development. Regional Studies, 53(11): 1509-1519.

Lerner, J. 2009. Boulevard of broken dreams: Why public efforts to boost entrepreneurship and venture capital have failed and what to do about it. Princeton: Princeton University Press.

Lincoln, Y. S., \& Guba, E. G. 1985. Naturalistic Inquiry. London, England: SAGE Publications, Ltd.

Locke, K. 2003. Grounded Theory in Management Research. 6 Bonhill Street, London EC2A 4PU: SAGE Publications, Ltd.

Lounsbury, M., \& Glynn, M. A. 2019. Cultural Entrepreneurship: A New Agenda for the Study of Entrepreneurial Processes and Possibilities (1st ed.). Cambridge University Press.

Marquis, C., \& Battilana, J. 2009. Acting globally but thinking locally? The enduring influence of local communities on organizations. Research in Organizational Behavior, 29: 283-302.

Mathias, B. D., Huyghe, A., Frid, C. J., \& Galloway, T. L. 2017. An identity perspective on coopetition in the craft beer industry. Strategic Management Journal, 39(12): 3086-3115. 
Nag, R., Corley, K. G., \& Gioia, D. A. 2007. The Intersection of Organizational Identity, Knowledge, and Practice: Attempting Strategic Change via Knowledge Grafting. Academy of Management Journal, 50(4): 821-847.

Patvardhan, S. D., Gioia, D. A., \& Hamilton, A. L. 2015. Weathering a Meta-Level Identity Crisis: Forging a Coherent Collective Identity for an Emerging Field. Academy of Management Journal, 58(2): 405-435.

Pauwels, C., Clarysse, B., Wright, M., \& Van Hove, J. 2016. Understanding a new generation incubation model: The accelerator. Technovation, 50-51: 13-24.

Polenske, K. 2004. Competition, Collaboration and Cooperation: An Uneasy Triangle in Networks of Firms and Regions. Regional Studies, 38(9): 1029-1043.

Porac, J. F., Thomas, H., \& Baden-Fuller, C. 1989. Competitive Groups as Cognitive Communities: The Case of Scottish Knitwear Manufacturers. Journal of Management Studies, 26(4): 397-416.

Romanelli, E., \& Khessina, O. M. 2005. Regional Industrial Identity: Cluster Configurations and Economic Development. Organization Science, 16(4): 344-358.

Roundy, P. T. 2017. Hybrid organizations and the logics of entrepreneurial ecosystems. International Entrepreneurship and Management Journal, 13(4): 1221-1237.

Saxenian, A. 1994. Regional advantage: Culture and competition in Silicon Valley and Route 128. Cambridge, Mass: Harvard University Press.

Schaeffer, V., \& Matt, M. 2016. Development of academic entrepreneurship in a non-mature context: The role of the university as a hub-organisation. Entrepreneurship \& Regional Development, 28(9-10): 724-745.

Schmidt, S. M., \& Kochan, T. A. 1977. Interorganizational Relationships: Patterns and Motivations. Administrative Science Quarterly, 22(2): 220-234.

Seale, C. 1999. Quality in Qualitative Research. Qualitative Inquiry, 5(4): 465-478.

Silverman, D. 2014. Interpreting qualitative data (Fifth edition). London: SAGE.

Spigel, B. 2017. The Relational Organization of Entrepreneurial Ecosystems. Entrepreneurship Theory and Practice, 41(1): 49-72.

Spigel, B., \& Harrison, R. 2018. Toward a process theory of entrepreneurial ecosystems: Toward a process theory of entrepreneurial ecosystems. Strategic Entrepreneurship Journal, 12(1): 151-168.

Steier, L., \& Greenwood, R. 2000. Entrepreneurship and the Evolution of Angel Networks. Organization Studies, 21(1): 163-192.

Syakhroza, M. A., Paolella, L., \& Munir, K. 2018. Holier than Thou? Identity Buffers and Adoption of Controversial Practices in the Islamic Banking Category. Academy of Management Journal, 1252-1277.

Thomas, L. D. W., \& Autio, E. Forthcoming. Innovation Ecosystems. In R. Aldag (Ed.), Oxford Research Encyclopaedia of Business and Management. UK: Oxford University Press.

Thompson, T. A., Purdy, J. M., \& Ventresca, M. J. 2018. How entrepreneurial ecosystems take form: Evidence from social impact initiatives in Seattle. Strategic Entrepreneurship Journal, 12(1): 96-116.

Tracey, P., Dalpiaz, E., \& Phillips, N. 2018. Fish out of Water: Translation, Legitimation, and New Venture Creation. Academy of Management Journal, 61(5): 1627-1666.

Tracey, P., \& Phillips, N. 2016. Managing the Consequences of Organizational Stigmatization: Identity Work in a Social Enterprise. Academy of Management Journal, 59(3): 740-765. 
Uzzi, B. 1997. Social Structure and Competition in Interfirm Networks: The Paradox of Embeddedness. Administrative Science Quarterly, 42(1): 35-67.

Van de Ven, A. H., Walker, G., \& Liston, J. 1979. Coordination Patterns Within an Interorganizational Network. Human Relations, 32(1): 19-36.

Van Maanen, J. 1979. The Fact of Fiction in Organizational Ethnography. Administrative Science Quarterly, 24(4): 539-550.

Vergne, J.-P., \& Wry, T. 2014. Categorizing Categorization Research: Review, Integration, and Future Directions. Journal of Management Studies, 51(1): 56-94.

Wickert, C., Vaccaro, A., \& Cornelissen, J. 2017. "Buying” Corporate Social Responsibility: Organisational Identity Orientation as a Determinant of Practice Adoption. Journal of Business Ethics, 142(3): 497-514.

Wright, M., Siegel, D. S., \& Mustar, P. 2017. An emerging ecosystem for student start-ups. The Journal of Technology Transfer, 42(4): 909-922.

Wry, T., Lounsbury, M., \& Glynn, M. A. 2011. Legitimating Nascent Collective Identities: Coordinating Cultural Entrepreneurship. Organization Science, 22(2): 449-463.

Zuckerman, E. W. 1999. The Categorical Imperative: Securities Analysts and the Illegitimacy Discount. American Journal of Sociology, 104(5): 1398-1438.

FIGURE 1

Theoretical Model of Ecosystem Interaction Pattern Emergence

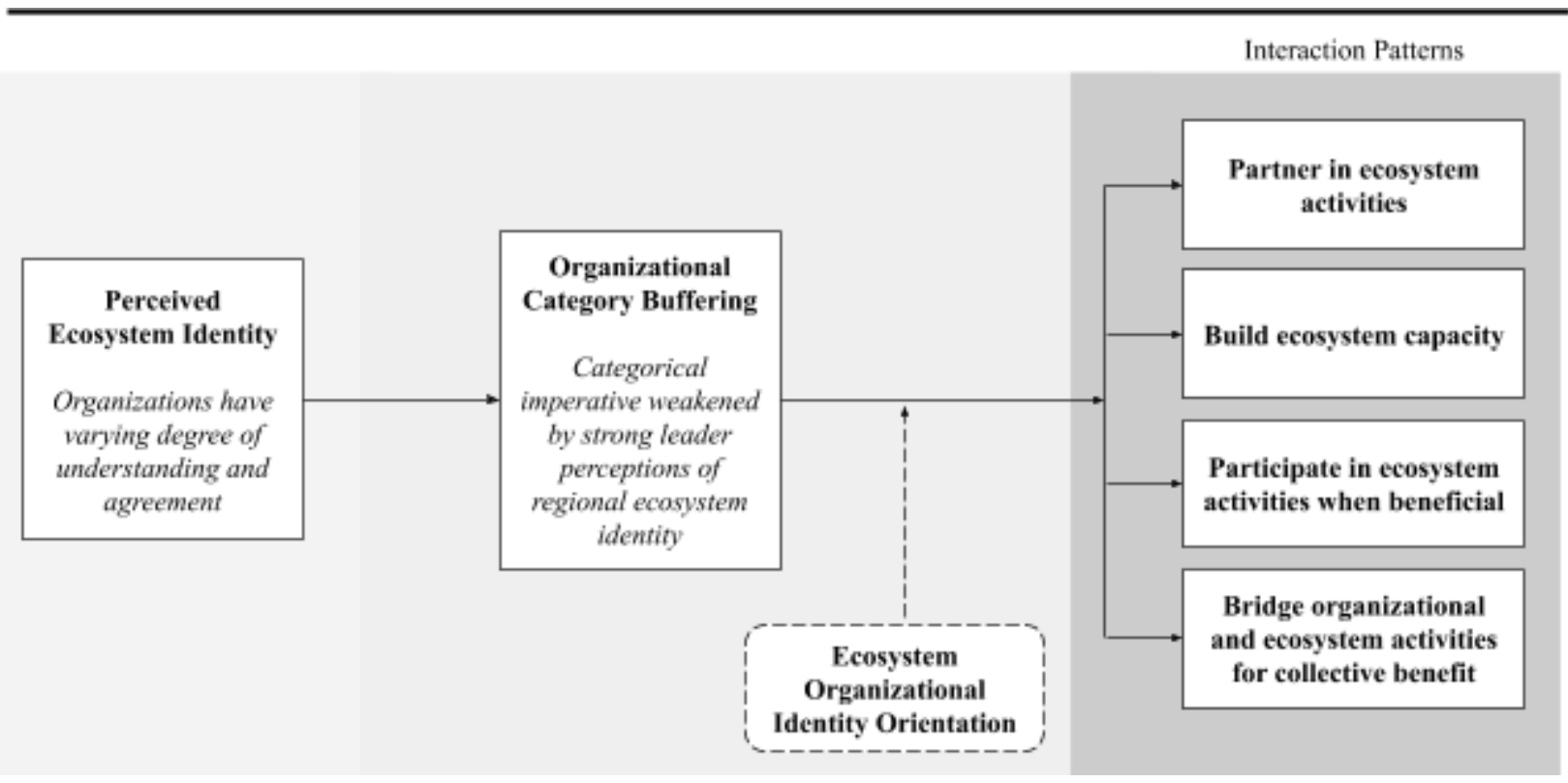


FIGURE 2

\section{Typology of Organizational Sponsorship Interaction Patterns}

\section{Ecosystem Organizational Identity Orientation}

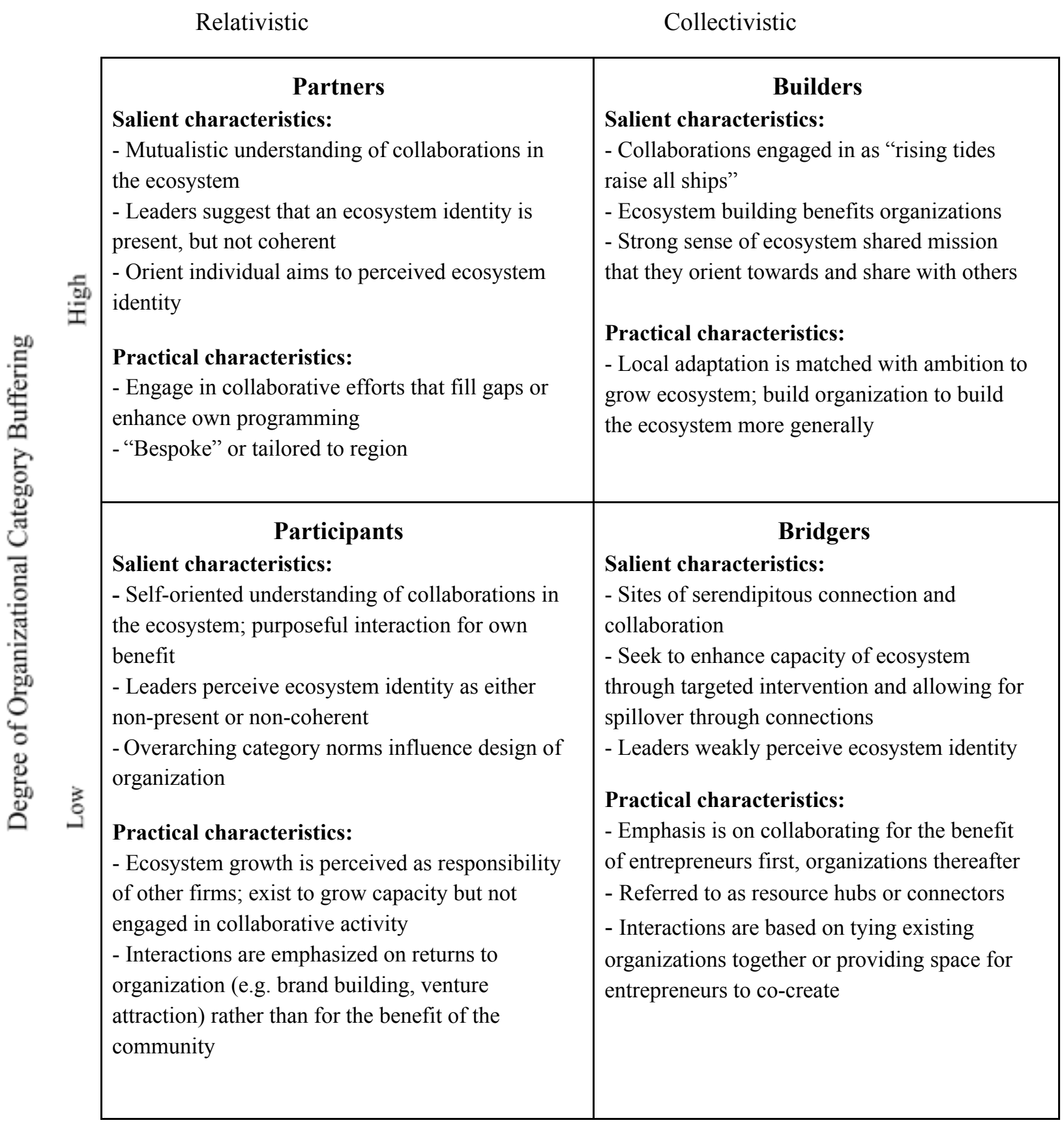


TABLE 1

Data Inventory

\begin{tabular}{|c|c|c|c|c|}
\hline \multicolumn{5}{|l|}{ Interviews } \\
\hline Pseudonym & Organization Type & Interaction Pattern & Role Type & Length \\
\hline Iota & Incubator & Partner & Business Advisor & $66 \mathrm{~min}$ \\
\hline $\mathrm{Xi}$ & Incubator & Builder & Director & $65 \mathrm{~min}$ \\
\hline Alpha & Accelerator & Builder & Director & $57 \mathrm{~min}$ \\
\hline Gamma & Workspace & Bridger & Board Member & $78 \mathrm{~min}$ \\
\hline Atom & Incubator & Participant & Manager & $54 \mathrm{~min}$ \\
\hline Sigma & Student Organization & Partner & President & $45 \mathrm{~min}$ \\
\hline Psi & Student Organization & Partner & Past President & $68 \mathrm{~min}$ \\
\hline Beta & Business Network & Builder & $\mathrm{CEO}$ & $53 \mathrm{~min}$ \\
\hline Chi & Venture Capital & Participant & Director & $44 \mathrm{~min}$ \\
\hline $\mathrm{Pi}$ & Incubator & Participant & CEO & $55 \mathrm{~min}$ \\
\hline Eta & Academic & Bridger & Director & $95 \mathrm{~min}$ \\
\hline Rho & Academic & Builder & $\mathrm{CEO}$ & $53 \mathrm{~min}$ \\
\hline Delta & Accelerator & Participant & Director & $45 \mathrm{~min}$ \\
\hline Omega & Workspace & Bridger & Board Member & $58 \mathrm{~min}$ \\
\hline Tau & Angel Capital & Participant & Director & $55 \mathrm{~min}$ \\
\hline \multicolumn{5}{|l|}{ Observation } \\
\hline \multicolumn{2}{|c|}{$\begin{array}{l}\text { Cambridge University Enterprise } \\
\text { Network Meeting }\end{array}$} & $\begin{array}{l}\text { Meeting of key stakeholders in } \\
\text { Cambridge entrepreneurial } \\
\text { ecosystem to share initiatives } \\
\text { and discuss shared activity }\end{array}$ & February 2019 & $120 \mathrm{~min}$ \\
\hline \multicolumn{2}{|c|}{ Enterprise Tuesday } & $\begin{array}{l}\text { Weekly event and gathering of } \\
\text { entrepreneurs, support } \\
\text { organizations }\end{array}$ & February 2019 & $120 \mathrm{~min}$ \\
\hline
\end{tabular}

Industry \& Practitioner Publications

\begin{tabular}{ll}
\hline The Cambridge Phenomenon & $\begin{array}{l}\text { Details history of Cambridge } \\
\text { cluster over the past 50 years }\end{array}$ \\
$\begin{array}{l}\text { TechNation reports; NESTA reports; } \\
\text { Global Accelerator Learning }\end{array}$ & $\begin{array}{l}\text { Used to sensitize to external } \\
\text { perspectives of the Cambridge } \\
\text { and UK ecosystems. }\end{array}$ \\
$\begin{array}{l}\text { Accelerator Network report } \\
\begin{array}{l}\text { Entrepreneurial support } \\
\text { organization annual reports }\end{array}\end{array}$ & $\begin{array}{l}\text { Used to develop understanding } \\
\text { of organization aims and } \\
\text { metrics. }\end{array}$
\end{tabular}

